\title{
THE SMOOTHNESS OF THE ELASTIC-PLASTIC FREE BOUNDARY OF A TWISTED BAR ${ }^{1}$
}

\author{
LUIS A. CAFFARELLI AND NESTOR M. RIVIERE
}

\begin{abstract}
We establish the regularity of the elastic-plastic free boundary of a multiply connected twisted bar. The regularity of the free boundary is determined by the regularity of the boundary of the domain.
\end{abstract}

The purpose of this note is to show how the results developed in [2] apply to the problem of a twisted bar and yield the regularity of the free boundary.

The study of the problem from the variational inequalities point of view was considered by T. Ting in several papers (see [3] for a complete bibliography) and by Brezis and Stampacchia [1]. The problem is as follows:

Let $D$ be a domain in $R^{2}$ verifying:

(a) $D$ has finite connectivity and is bounded internally by Jordan curves $\Gamma_{i}$ and externally by a Jordan curve $\Gamma_{0}$.

(b) Each $\Gamma_{i}$ is composed of a finite number of $C^{2}$ arcs (up to the endpoints) on each of which the curvature $k$ assumes only a finite number of maxima and minima. Let $G_{j}$ be the domain enclosed by $\Gamma_{j}$.

We consider the following problem.

Problem 1. Let $K$ be the convex subset of $H_{0}^{1}\left(G_{0}\right)$ :

$$
K=\left\{u: u \in H_{0}^{1}, u=\text { constant a.e. on } G_{j}, j>0,|\nabla u| \leqslant 1 \text { a.e. }\right\} \text {. }
$$

Find $u$ on $K$ such that $\int_{G_{0}} \nabla u \nabla(v-u) d x \geqslant \int_{G_{0}} \mu(v-u) d x$ for any $v$ on $K(\mu$ a positive constant).

Problem 1 is known to have a unique solution in $H_{0}^{1}\left(G_{0}\right)$ (see [1]).

Let $u$ be a solution of Problem 1 and $C_{i}=u / G_{i}$. (Note that $\left|C_{i}-C_{j}\right|$ $\leqslant d\left(G_{i}, G_{j}\right)$.) Consider

$$
\begin{aligned}
& F_{1}(x)=\min _{j \geqslant 1}\left[C_{i}+d\left(x, G_{i}\right), d\left(x, e G_{0}\right)\right], \\
& F_{2}(x)=\max \left[C_{i}-d\left(x, G_{i}\right)\right]
\end{aligned}
$$

Then there exists a unique solution $u^{*}$ to the problem.

Problem 2. Find $u^{*}$ belonging to $K_{2}=\left\{v: v \in H_{0}^{1}(G)\right\}, F_{1} \leqslant v \leqslant F_{2}$ such that $\int \nabla u^{*} \nabla\left(v-u^{*}\right) \geqslant \int \mu\left(v-u^{*}\right)$.

Received by the editors May 5, 1976.

AMS (MOS) subject classifications (1970). Primary 35A15; Secondary $73 E 99$.

${ }^{1}$ Research supported in part by NSF Grant 7406375A01. 
It has been proved [3] that the two problems are equivalent and that the solution is locally in $W^{2, p}$ inside $D$. When the boundary is sufficiently smooth (say $C^{3}$ ) and the domain simply connected, this result was proved by Brezis and Stampacchia [1]; in the general case, the result was obtained by Ting [3]. The important consequence of this fact, which is essential for the presentation of this note, is that it assures us that the free boundary does not touch the ridge of the domain in the interior of $D$. As Ting points out [3], the free boundary is parametrized in a unique way by arcs of the boundary.

We intend to prove that the local regularity of the free boundary depends only on the local regularity of the corresponding portion of the boundary that parametrizes it.

For simplicity we will remove from our discussion the family of segments where $F_{1}$ could equal $F_{2}$ and the boundary of the regions corresponding to reentrant corners. Although across such parts of $D$, the solution, $u$, preserves its local $C^{1,1}$ regularity, we will analyze these cases elsewhere.

In the remaining parts let us notice that on the coincidence set (and hence away from the ridge) the majorant $\Phi$ has the same smoothness as that of the piece of boundary where the distance is attained or is analytic in the region corresponding to a reentrant corner.

Furthermore, the only cases in which $\Delta \Phi<0$ correspond to pieces of boundary with strictly positive curvature (hence outside the regions corresponding to reentrant corners). It is easy to check that there $\nabla(\Delta \Phi)$ never vanishes $\left(\Delta \Phi=-1 /\left(k^{-1}-\Phi\right)=-k /(1-k \Phi)\right)$.We can now easily deduce the following.

THEOREM. Let $\left(x_{0}, y_{0}\right)$ belong to the free boundary, and let $\sigma\left(x_{0}, y_{0}\right)$ be the boundary point where $\left(x_{0}, y_{0}\right)$ realizes its distance to the boundary. Then:

(a) If in a neighborhood of $\sigma$ the boundary is of class $C^{k, \alpha}$ (analytic), then in a neighborhood of $\left(x_{0}, y_{0}\right)$ the free boundary is of class $C^{k-1, \alpha}$ (analytic) with a continuous tangent $(k \geqslant 3)$.

(b) If $\sigma\left(x_{0}, y_{0}\right)$ is the vertex of a reentrant corner, then in a neighborhood of $\left(x_{0}, y_{0}\right)$ the free boundary is analytic.

Proof. From Theorem 1 of [2], parts (a) and (b) follow except for the possible presence of isolated quadratic cusps entering the noncoincidence set.

We will show that there are no cusps.

In fact, if $\eta$ denotes the direction of the segment joining $\left(x_{0}, y_{0}\right)$ and $\sigma\left(x_{0}, y_{0}\right)$, which must be the axis of the cusp, $u_{\eta \eta}$ is continuous on the noncoincidence set up to the free boundary in a neighborhood of the cusp. Because $(u-\Phi)_{\eta \eta}=\frac{1}{2}(\Delta(u-\Phi))(\nu \circ \eta)^{2}$ on the free boundary whenever the free boundary has a normal vector $\nu$, and that converges to zero when the boundary point approaches the curve.

But $\Phi_{\eta \eta}\left(x_{0}, y_{0}\right)=0$, therefore $u_{\eta \eta}\left(x_{0}, y_{0}\right)=0$. On the other hand, $u_{\eta}$ is harmonic in the noncoincidence set, $\left|u_{\eta}\right| \leqslant 1$ and $u_{\eta}\left(x_{0}, y_{0}\right)=\Phi_{\eta}\left(x_{0}, y_{0}\right)$ $=1$. Hence, by the strict maximum principle (note that a ball can be placed 
on the noncoincidence set), $u_{\eta}<0$, a contradiction.

\section{REFERENCES}

1. H. R. Brezis and G. Stampacchia, Sur la régularité de la solution d'inéquations elliptiques, Bull. Soc. Math. France 96 (1968), 153-180. MR 39 \#659.

2. L. A. Caffarelli and N. M. Rivière, Smoothness and analyticity of free boundaries in variational inequalities, Ann. Scuola Norm. Sup. Pisa Sci. Fis. Mat. (4) 3 (1976), 289-310.

3. T. W. Ting, Elastic plastic torsion problem over multiply connected domains (to appear).

School of Mathematics, University of Minnesota, Minneapolis, Minnesota 55455 